\title{
Radionuclide evaluation of the compensatory capacity of the kidneys
}

\begin{abstract}
Aim: Assess the compensatory capacity of the kidneys on the basis of determining the volume of functioning kidney tissue according to single-photon emission scintigraphy (SPECT).

Materials and methods: Our study included 65 patients with kidney parenchyma cancers, 32 patients ones had cysts and 20 patients without kidney pathology. Besides we investigated 57 children with different no cancers pathology. The following parameters were used: the ratio of the volumes of functioning tissue and the specific activities between the affected and contralateral kidneys and the ratio of the values of the obtained kidney volumes to the appropriate volume for the patient under study, depending on the data of his anthropometry (sex and age).
\end{abstract}

Results: Based on the results obtained, the maximum compensatory renal reserve occurs in the case of a decrease in the volume of functioning tissue of the affected kidney and a simultaneous increase in the volume of the contralateral kidney. In the presence of ratios of the affected kidney volume with the tumor and contralateral $<0.65$ and the ratio of their specific activities $<0.75$, the cases are classified as tumors with the presence of infiltrating growth. In other cases, objects are referred to tumors without infiltrating growth.

Conclusion: Thus, the proposed integral parameters allow assessing the compensatory potential of the kidneys. The value of the functional volume according to SPECT, allows to get important information about the state of functioning parenchyma of the kidney. The data used in the selection of indications for conserving surgery.

Keywords: SPECT, functional volume, compensatory capacity of the kidneys
Volume 6 Issue 5 - 2018

\author{
VVidioukov,' O Bessolova,' N Gerasimova² \\ 'Nuclear medicine Department, Russian Medical Academy of \\ Postgraduate Education, Russia \\ ${ }^{2}$ Radiological department, Scientific Centre of Children's Health, \\ Russia
}

Correspondence: Vladimir Vidioukov, 2-nd Botkinskiy pr.,7 Moscow, I25284, Russia, Tel +7 90351501 06,

Email viduk32@yandex.ru

Received: August 30, 2018 | Published: October 01, 2018

\section{Introduction}

Most often radionuclide methods in urology are used to assess the functional abilities of the kidneys on the basis of dynamic studies and detection of foci on scintigrams using planar gamma cameras or on tomoscintigraphic sections using a single-photon emission computer tomograph (SPECT). Significantly less commonly used is the method of determining the volume of functioning organ tissue by summing the volume elements that form the image of the organ. This work is based on the method we developed for determining the functioning of kidney tissue using SPECT. ${ }^{1,2}$ Our studies have shown that with SPECT, it is possible to accurately determine the volume of functioning organ tissue and on its basis to determine integral parameters that ensure high accuracy of diagnosis. Phantom studies have shown that for organs such as the kidney with a fixed background cutoff, the error in calculating the volume does not exceed 5.1\%.

The purpose of this work is to assess the compensatory capacity of the kidneys using the values of the volume of functioning kidney tissue obtained on the basis of radionuclide methods.

\section{Materials and methods}

Radionuclide studies were performed in adult patients using SPECT. In this study, we analyzed 97 patients (65 patients had malignant kidney tumors, 32 patients had cysts, a control group of 20 patients without pathological changes in the kidneys).

In addition to radionuclide studies, all patients underwent ultrasonic, x-ray, clinical, laboratory and morphological studies. Out of 65 patients with unilateral malignant lesions, 40 cases without infiltrating growth and 25 cases with infiltrating growth of the focus.

When processing the SPECT data, the volume of functioning tissue of the right and left kidneys was calculated.

In addition, radionuclide studies of 51 children aged 1-17 years were analyzed, in which the kidney volume was determined on the basis of static polypositional scintigraphy calculating the volume modeling the ellipse. Of these, 12 patients were diagnosed with hydronephrosis, 12 megaurether, and 27 vesicoureteral reflux. Studies were performed 6 months after surgery. To conduct radionuclide studies, the radiopharmaceutical DMSA was used in accordance with the dosage standards.

When processing radionuclide studies, the following values were determined: $\mathrm{Vl}, \mathrm{Cl}, \mathrm{Vr}, \mathrm{Cr}$, where $\mathrm{Vl}$ is the volume of the left kidney volume in $\mathrm{cm}^{3}, \mathrm{Cl}$ is the value of the total activity of the left kidney (imp), $\mathrm{Vr}$ is the volume of the right kidney in $\mathrm{cm}^{3}, \mathrm{Cr}$ is the sum activity of the right kidney (imp).

To estimate the data on the basis of the values obtained, the following integral indicators are proposed: Vaff / Vcont; (Caff / Vaff): (Ccont / Vcont); where Vaff - the volume of the affected kidney, Vcont - the volume of the contralateral kidney, Caff / Vaff - specific activity of the affected kidney, Ccont / Vcont - specific activity of the contralateral kidney.

The question of which kidney is affected was solved based on the data of a comprehensive diagnostic examination. In addition to these parameters, we used indicators that characterize the ratios of 
calculated kidney volume to the proper for the patient: Vaff / Vnorm; Vcont / V norm. According to radionuclide studies of the control group, the maximum kidney volume (Vnorm) occurs in men for 2535 years and decreases in other age ranges. ${ }^{3}$

Based on this situation and using the correction factor for each patient, you can determine the value of the proper volume by the formula:

Vnorm $=$ Vnorm $*: \mathrm{Kk}$, where $\mathrm{Kk}$ is the correction factor, which is set according to the dependence of the weight of the kidney on sex and age. ${ }^{4}$ Note that the ratios of the calculated volumes of the kidneys to the proper can be determined using various diagnostic methods: ultrasound, $\mathrm{CT}$ and others.

\section{Results}

Table 1 presents the results of statistical processing of integrated indicators. Considering the results obtained on the proposed parameters, the following regularities can be noted.

Table I Statistical indices for various clinical groups with SPECT kidney

\begin{tabular}{lllll}
\hline & $\begin{array}{l}\text { Vaff/ } \\
\text { Vnorm }\end{array}$ & $\begin{array}{l}\text { Vcont/ } \\
\text { Vnorm }\end{array}$ & $\begin{array}{l}\text { Vaff/ } \\
\text { Vcont }\end{array}$ & $\begin{array}{l}\text { (C/Vaff)/ } \\
\text { (C/V)cont }\end{array}$ \\
\hline Norm & & & $1.0 \pm 0.02$ & $0.96 \pm 0.02$ \\
$\begin{array}{l}\text { Cyst } \\
\begin{array}{l}\text { Tumor without } \\
\text { inf. growth }\end{array}\end{array}$ & $1.166 \pm 004$ & $1.08 \pm 004$ & $1.0 \pm 0.07$ & $0.98 \pm 004$ \\
$\begin{array}{l}\text { Tumor with inf. } \\
\text { growth }\end{array}$ & $0.93 \pm 0.09$ & $\mathrm{I} .54 \pm 0.06$ & $0.73 \pm 0.08$ & $0.71 \pm 007$ \\
\hline
\end{tabular}

Parameters for a group of patients with cysts do not differ significantly from the control group. In cases of tumors with no infiltrating growth, there is a slight decrease in the volume of the affected kidney in relation to the contralateral $(0.88)$, while maintaining the level of specific activity (0.99). At the same time, the size of the affected kidney increases with respect to the proper volume (1.16) along with the compensatory increase in the contralateral kidney. In cases of tumors with the presence of infiltrating growth, the volume of the affected kidney decreases with respect to the contralateral kidney to 0.73 , together with a decrease in the ratio of specific activities to 0.71 . The decrease in these indicators is due to a decrease in the functioning of volumetric elements outside lesions. The loss of the volume of functioning tissue with infiltrating growth of the tumor leads to a decrease in the specific activity of the affected kidney and, as a result, an increase in these parameters for the contralateral kidney. On the basis of the parameters Vaff / Vcont and (Caff/ Vaff): (Ccont/ Vcont), two classes of objects can be distinguished - characteristic of tumors with and without infiltrating growth. The following decisive rule is used: objects having the ratio of the volume of the affected kidney to the volume of the contralateral kidney $<0.65$ and the ratio of the specific activities of the affected and contralateral kidneys $<0.75$ belong to the class characteristic of tumors with the presence of infiltrating growth (Figure 1). In other cases, objects belong to a class that is characteristic of tumors without infiltrating growth (Figure 2). With this method, the reliability of the test increases with the size of the focus. The accuracy of this method for the entire range of tumor sizes (from 2.5 to $9 \mathrm{~cm}$ ) is $83.1 \%$, sensitivity is $75.8 \%$ and specificity is $90.1 \% .^{5}$

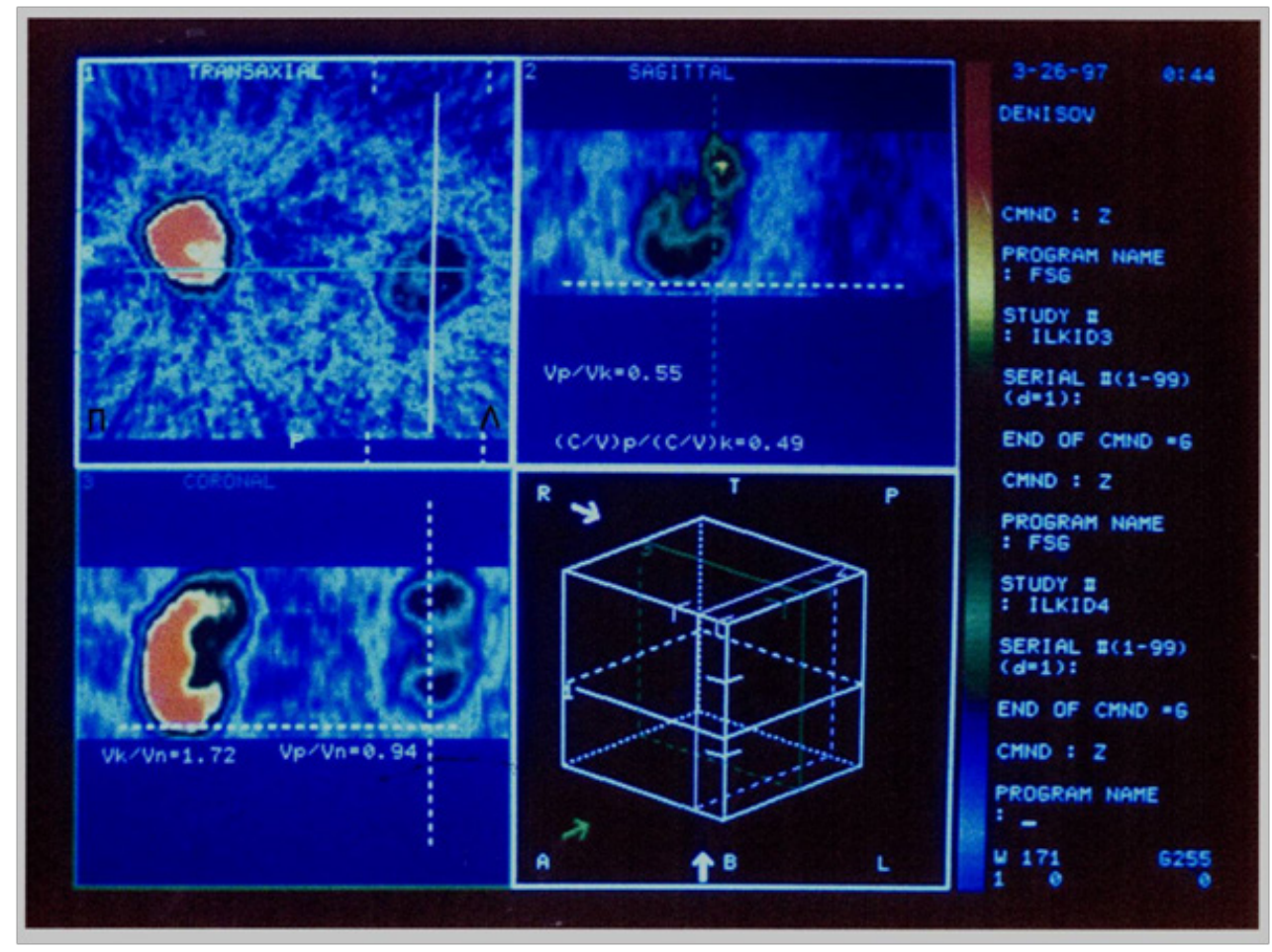

Figure I SPECT in case of left kidney cancer with infiltrating growth.

Vaff $/$ cont $=0.55 ;(C / V)$ aff $/(C / V)$ cont $)=0.49 ;$ Vkont $/$ Vnorm $=1.72$ 


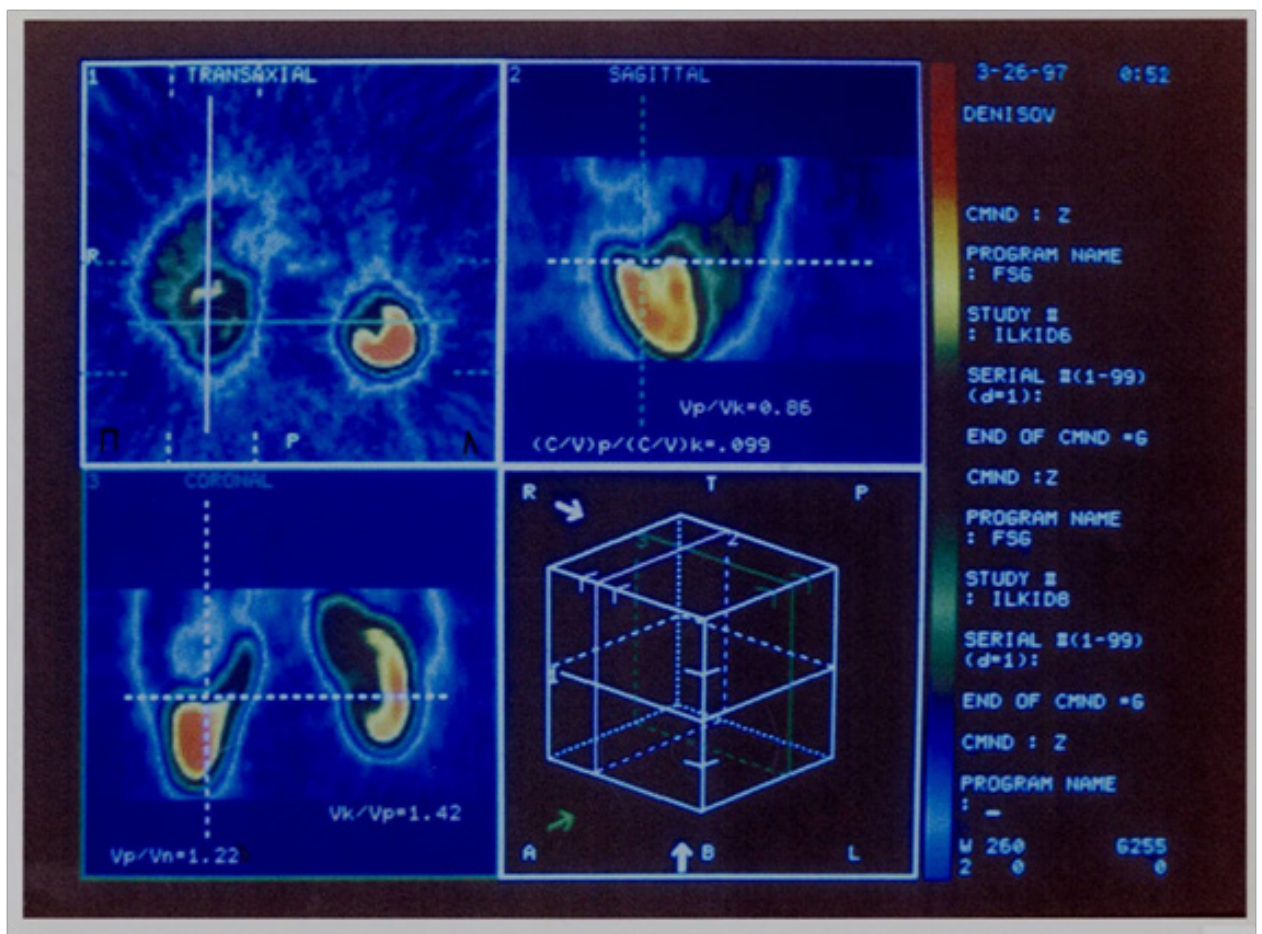

Figure 2 SPECT with cancer in the upper segment of the right kidney with no infiltrating growth.

Vaff $/$ V cont $=0.85 ;(\mathrm{C} / \mathrm{V})$ aff $/(\mathrm{C} / \mathrm{V})$ cont $)=0.99 ; \mathrm{Vkont} /$ Vnorm $=1.22$

In addition to these parameters, the indicators characterizing the ratios of the allocated volumes of the kidneys to the proper for the examined patient, according to his age and sex, were used. In the focus of the renal cell carcinoma less than $3 \mathrm{~cm}$ in diameter in the parenchyma of the affected and contralateral kidneys, no noticeable functional changes are detected. The volume of the kidneys within the age and sex norms. A slight loss of the volume of the parenchyma of the affected kidney with lesions less $3 \mathrm{~cm}$ is compensated for by including nephrons that make up the functional reserve of the kidneys. With a focus larger than $3 \mathrm{~cm}$, an increase in the volume of functional tissue is observed. There is depletion of the functional reserve of the renal parenchyma and for the preservation of homeostasis, the processes of hypertrophy of existing nephrons are included. Both kidneys react with symmetrical compensatory hypertrophy as a single organ.

The next stage in the development of the compensatory response occurs when tumors exceed $6 \mathrm{~cm}$. The reserves of the affected kidney are exhausted; the volume of its functional tissue (Vaff) begins to decrease. At this stage, most often the germination of tumor pseudocapsules and the acquisition of a lesions infiltrating growth The volume of the functional tissue of the contralateral kidney (Vcont) reaches a maximum level exceeding the average on 54\% proper volume. The largest excess of the size of the contralateral kidney in relation to the proper volume was $72 \%$ (Figure 1).

Therefore, the degree of compensatory response of the parenchyma of the affected and contralateral kidney and comparing them with each other, as well as with the proper volume of the kidneys of the patient being examined, can provide data on the growth of the tumor lesion, predict the level of compensatory hypertrophy. This approach is useful for assessing the functional parameters of the parenchyma after surgical treatment (nephrectomy and resection of the kidney with a tumor) at 6 and 12 months. As a result of a repeated SPECT study in patients after nephrectomy, it is possible to compare the volume of the contralateral kidney with the data prior to surgery. There is an increase in the volume of the contralateral kidney relative to that due 6 months after surgery, an average of $69 \%$. Further increase in the volume of the contralateral kidney (after 12 months) is not observed. As a result of resection of compensatory hypertrophy, the contralateral kidney is exposed (on average 54\%).

Table 2 shows the statistical integrated indicators for different clinical groups, as well as data from patients without kidney damage in children. Studies were performed 6 months after surgery. Consider changes in these indicators for different clinical groups.

Table 2 Statistical indices for various clinical groups for radionuclide studies of kidneys in children

\begin{tabular}{lllll}
\hline & $\begin{array}{l}\text { Vaff/ } \\
\text { Vnorm }\end{array}$ & $\begin{array}{l}\text { Vcont/ } \\
\text { Vnorm }\end{array}$ & $\begin{array}{l}\text { Vaff/ } \\
\text { Vcont }\end{array}$ & $\begin{array}{l}\text { (C/Vaff)/ } \\
(\mathbf{C} / \text { V)cont }\end{array}$ \\
\hline Norm & & & $1.0 \pm 0.02$ & $0.96 \pm 0.02$ \\
Hydronephrosis & $1.07 \pm 0.12$ & $1.22 \pm 0.07$ & $1.0 \pm 0.15$ & $0.65 \pm 0.13$ \\
Megaureter & $0.53 \pm 0.11$ & $1.21 \pm 0.2$ & $0.51 \pm 0.14$ & $0.86 \pm 0.08$ \\
VUR & $0.64 \pm 0.13$ & $1.05 \pm 0.09$ & $0.71 \pm 0.14$ & $0.88 \pm 0.03$
\end{tabular}


Hydronephrosis: Among the patients with this diagnosis, two groups can be distinguished. The main and first group; A definite decrease in the specific activity of the affected kidney in relation to the contralateral kidney, the volume of the affected kidney is not reduced in relation to the proper; the volume of the contralateral kidney corresponds to the proper. The second group; A sharp decrease in the specific activity of the affected kidney in relation to the contralateral $(0.11-0.12)$, the correspondence of the affected volume to the due $(0.94-0.98)$ and an increase in the volume of the contralateral kidney in relation to the proper. (1.36-1.5), which is explained by a high level of compensatory inclusion of reserve nephrons.

Megaureter: In the majority of patients in this group, there is a significant decrease in the volume of the affected kidney in relation to the proper. In a number of patients, this decrease is accompanied by a significant decrease in the ratio of the specific activities of the affected and contralateral kidneys and a sharp increase in the volume of the contralateral kidney relative to the proper (up to 1.7).

Vesicoureterall reflux(VUR). The most pronounced change is a decrease in the volume of the affected kidney with insignificant changes in the ratio of specific activities and the size of the contralateral kidney. Thus, the proposed indicators are sensitive for detecting the extent of the lesion and evaluating the compensatory possibilities for radionuclide studies of kidneys in children.

In Figure 3 shows an example of an 11 year old patient with hydronephrosis of the left kidney. Integral indicators have changes with respect to corresponding to the data for the group norm, which indicates a stable phase of the compensatory process. Figure 4 shows an example of a 17 year old patient who also has hydronephrosis of the left kidney. The values of the integral indicators are significantly changed in relation to the data of the group norm, which indicates the maximum possibilities of the compensatory process.

Summarizing the results obtained for clinical groups, the following stages of compensatory processes in the kidneys can be distinguished.

1) Change in the volume of the affected kidney in relation to the proper with minor changes in other parameters.

2) Reduction of the ratio of specific activities of the affected and contralateral kidney with minor changes in other parameters.

3) Significant decrease in the ratio of specific activities of the affected and contralateral kidneys with a sharp increase in the volume of the contralateral kidney in relation to the proper (up to 1.72) and a decrease in the volume of the affected kidney.

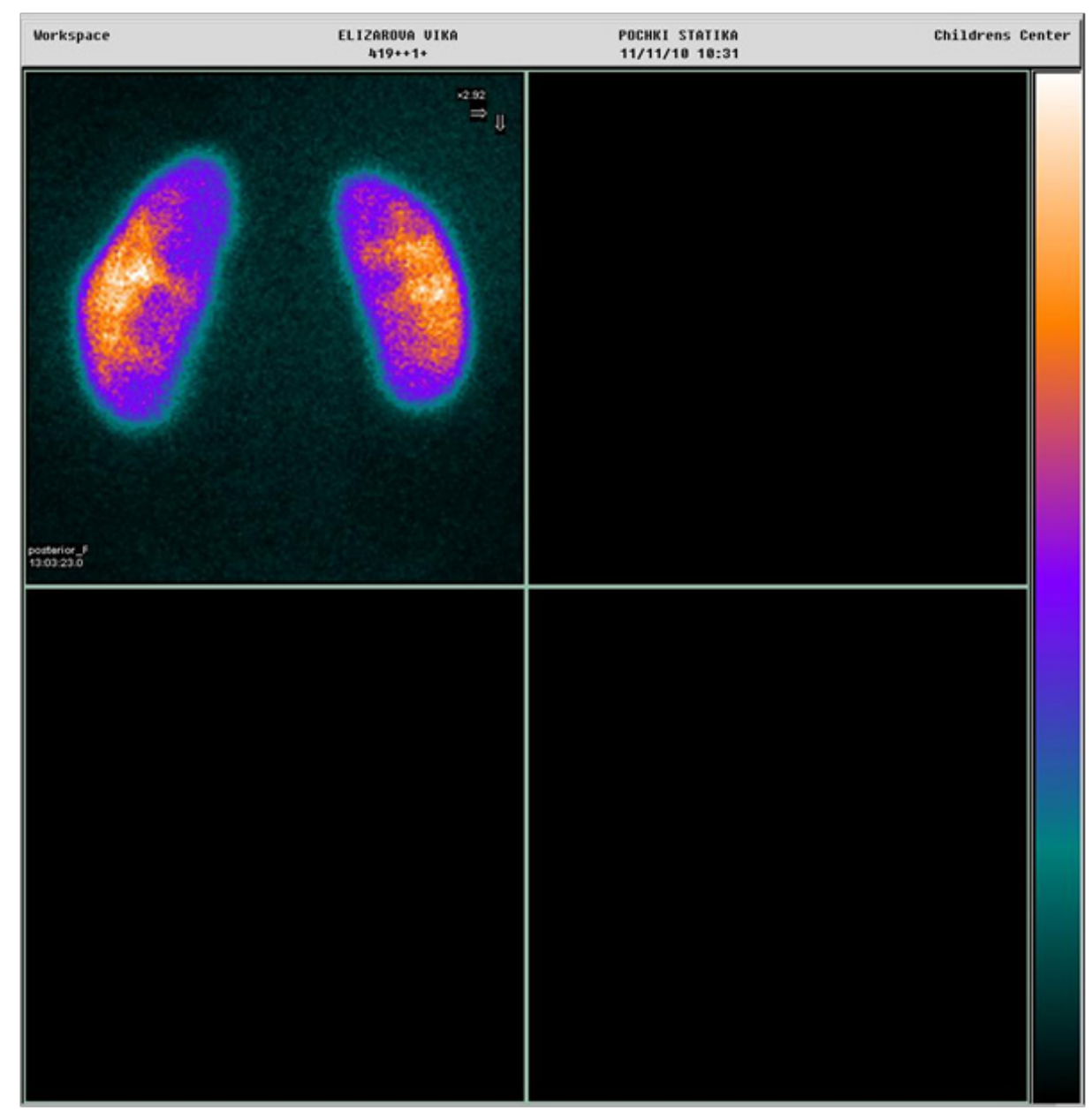

Figure 3 Multiplanar kidney study of an II-year-old female patient with hydronephrosis of the left kidney.

Vdam $/$ Vcont $=1.38,(\mathrm{C} / \mathrm{V}) \mathrm{dam} /(\mathrm{C} / \mathrm{V})$ cont $)=\mathrm{I} .14$. V cont $/$ norm $=1.24$ 


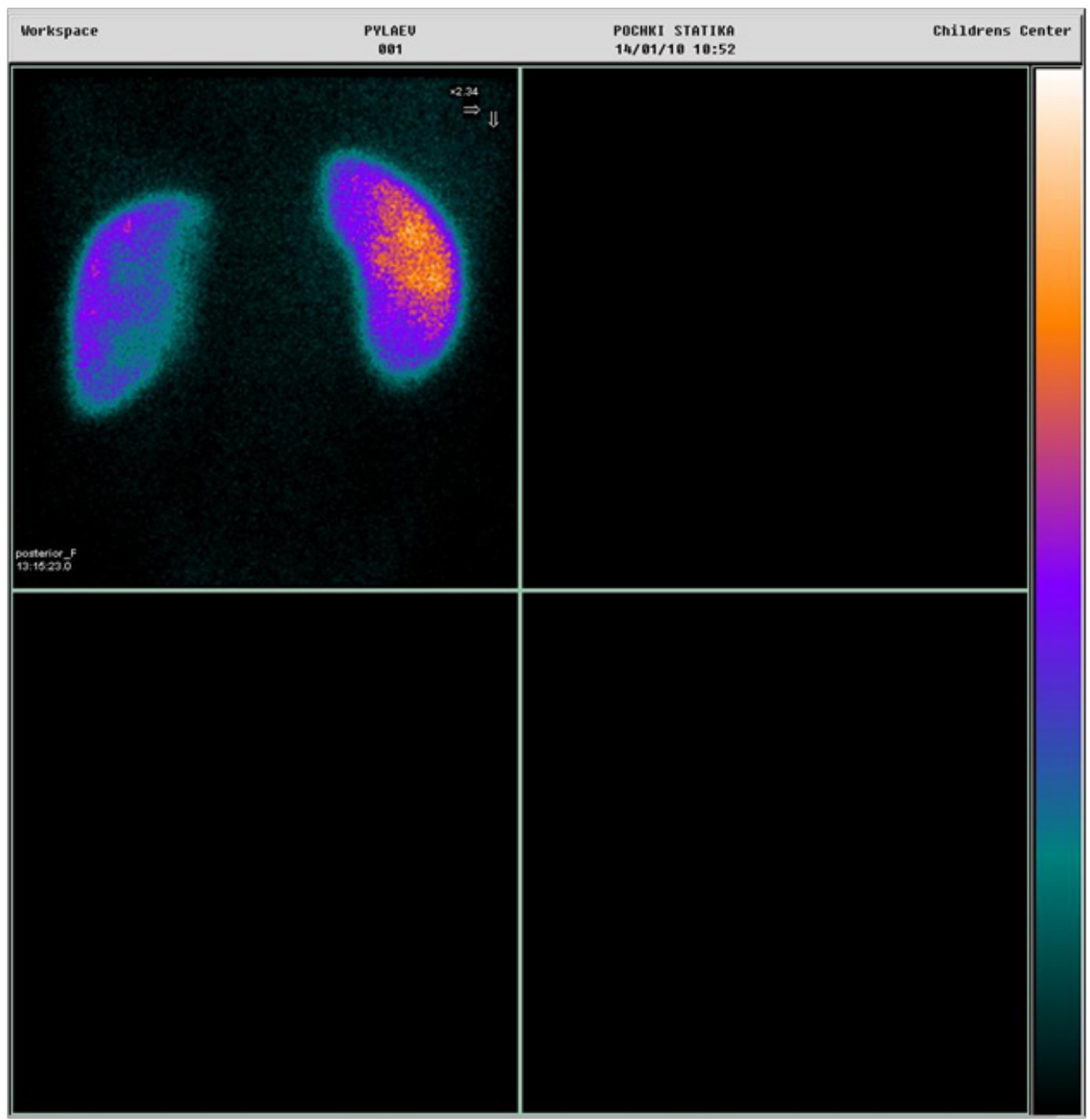

Figure 4 Multiplanar kidney study of a 17-year-old patient with hydronephrosis of the left kidney.

Vdam $/$ Vcont $=0.86,(C / V)$ dam $/(C / V)$ cont $)=0.66$, Vcont $/$ norm $=1.47$

\section{Summary}

Thus, conducted studies of adult and pediatric patients with various kidney lesions showed that using parameters based on determining the volume of functioning kidney tissue it is possible to evaluate the compensatory possibilities of the parenchyma of the affected and contralateral kidneys with the help of SPECT or planar radionuclide studies. Identification of the peculiarities of the changes in the anatomical and functional state of the parenchyma allows us to approach objectively the choice of tactics of surgical intervention and treatment, and dynamic monitoring of urological patients.

\section{Acknowledgements}

None.

\section{Conflict of interest}

Author declares that there is no conflict of interest.

\section{References}

1. Vidyukov VI, Stepanov VN. The method of diagnosis of kidney tumors. Patent No; RU94041974A, 1997.

2. Vidyukov VI. Assessment of medical images. Monograph M. 2004

3. Gopala Rao UV, Wagner HM. Normal weights of human organs. Radiology. 1972;102(2):337-339.

4. Vidyukov VI. Determination of the volume of internal organs and its diagnostic value. J Medical alphabet. 2017;1(12);31-33.

5. Stepanov VN, Vidyukov VI, Seregin AV, et al. Change in anatomical and functional state of parenchyma in unilateral early renal cell carcinoma before and after surgical treatment. Urologiia. 2001;4:22-26. 